\title{
LA AVENTURA DEL CONOCIMIENTO DE ALEJANDRO LOSADA
}

The adventure of knowledge of Alejandro Losada

Hugo Herrera Pardo*

\section{DesVenturas DEL CONOCIMIENTO}

Dentro del intenso proceso de renovación que experimentó la crítica literaria latinoamericana a partir de la década de 1970, Alejandro Losada fue sin duda uno de sus más importantes animadores, ejecutando y promoviendo un proyecto de Historia Social de las Literaturas Latinoamericanas que, en su momento, permitió develar un completo campo de relaciones para el análisis e interpretación de tales literaturas, en conjunto con una actitud comprometida y colectiva en la tarea del crítico. Por tanto, no deja de ser paradójica la situación de que, treinta años después de su deceso, la bibliografía sobre su obra apenas esté integrada por un par de volúmenes, además de algunos artículos y capítulos de libro, y que inclusive, su trabajo no posea mayor circulación. En lo que sigue, desarrollaré una larga introducción sobre su tarea crítica con el objetivo de poder fundamentar una explicación para la circunstancia aludida.

\section{ALEJANDRO LOSADA: UNA INTRODUCCIÓN}

2.1 Trayectoria investigativa y tradición previamente dominante

En 1975, en la introducción del primero de una larga serie de elaborados trabajos $^{1}$ en los cuales comienza a desarrollar su sistemático modelo historiográfico de características hipotético-deductivas, Alejandro Losada enumera lo que él interpreta como las cinco principales limitaciones ${ }^{2}$ de los modelos (a los cuales califica de "arbitrarios, incoherentes o insuficientes") de historia literaria con los que se desempeñó la tradición crítica latinoamericana previamente dominante. Tras aquel brevísimo inventario, Losada realiza una distinción basada en el "tipo de discurso" entre la

\footnotetext{
${ }^{1}$ El trabajo aludido es "Los sistemas literarios como instituciones sociales en América Latina", aparecido en el № 1 de la Revista de Crítica Literaria Latinoamericana. Al año siguiente conformaría el tercer capítulo del primero de sus libros abocados a explicar su modelo de Historia social de las literaturas latinoamericanas: Creación y praxis. La producción literaria como praxis social en Hispanoamérica y el Perú (1976).

${ }^{2}$ Para Losada, los principales problemas teórico-metodológicas de la historia literaria latinoamericana previamente dominante obedecían al hecho de recaer en la simple enumeración de autores, en una ordenación según criterios que habían sido tomados de otras tradiciones literarias, en periodizaciones no pertinentes, en la consideración nacional de las historias literarias, en la inadecuada, o en algunos casos inexistente, articulación entre los fenómenos sociales y los fenómenos literarios, entre otras causas. Estableciendo una apresurada síntesis, estos cinco problemas teórico-metodológicos detectados por Losada podrían definirse como: a) Diferencialidad del desarrollo diacrónico subregional; b) Simultaneidad de fenómenos literarios diferentes; c) Incoherencia del modelo generacional, producto del problema anterior; d) Utilización de criterios descriptivos e interpretativos disímiles e impertinentes; y e) No distinción entre la valoración estética de una obra o conjunto de ellas y su significación social.
} 
tradición previa y su programa investigativo. Esta toma de posición puede leerse también como el envés de la trayectoria crítica completa del mismo Losada. Quiero decir, que, a la manera de un palimpsesto, esta toma de posición condensa tanto el antes como el durante de su proyecto de Historia Social de las Literaturas Latinoamericanas:

La crítica no ha trabajado con modelos, no ha formulado hipótesis que pudieran ser verificadas y discutidas y, más aún, no ha trascendido la etapa monográfica de la recopilación de datos y el estudio de autores y obras particulares. Los diversos trabajos sobre conjuntos se caracterizan por la ausencia de la reflexión teórica y epistemológica, utilizando el discurso ensayístico, las más de las veces simplificador y voluntarista. Esos ensayos, sin embargo, suponen un cierto modelo tácito de comprensión del fenómeno que podría ser formulado como una hipótesis de trabajo. Si se realizara este nuevo tipo de discurso, saldría a luz de una manera inmediata la insuficiencia de esos trabajos que no puede comprender sistemáticamente los hechos señalados. Este es el sentido de este modelo ( $L a$ Historia, 40).

He dicho antes y durante. En efecto, las primeras publicaciones en la trayectoria crítica $^{3}$ de Losada habían sido precisamente trabajos monográficos sobre José Hernández ${ }^{4}$, Ciro Alegría y José María Arguedas ${ }^{6}$. Según confiesa en el "Prólogo" a La literatura en la sociedad de América Latina. Perú y el Río de la Plata 1837-1880, la comparación entre estas monografias le había sugerido "la posibilidad de que los tres configuraban una misma tendencia literaria" (11). En su atención a los tres autores señalados anteriormente, Losada advirtió las evidentes limitaciones del objeto de estudio de la disciplina, en tanto que sus respectivas interpretaciones develaban una serie de regularidades que podrían permitir ampliar el objeto formal de análisis y, por ende, las explicaciones para el fenómeno en cuestión. En aquel "Prólogo", el crítico argentino menciona una extensa serie de elementos que Hernández, Alegría y Arguedas compartían, en el marco de su interpretación. Estas regularidades o elementos comunes se encontraban basados, en primer lugar, en las funciones sociales que pretendían cumplir aquellas obras en sus respectivas sociedades, a juicio de Losada: "la misma voluntad de configurar un ciclo histórico que incidía esencialmente en la situación del hombre popular; la intención de dar cuenta de la totalidad de la sociedad, configurándola en sus niveles más mediatos inaccesibles para la observación del sujeto

\footnotetext{
${ }^{3}$ Se hace necesario remarcar que se alude a su obra crítica sobre la literatura latinoamericana, ya que, y aunque se trate de un hecho aislado en su producción intelectual, Alejandro Losada publicó una novela a comienzos de la década del setenta, tras su dificil y breve experiencia (1968-1970) como asesor de gobierno durante el periodo de Juan Carlos Onganía. La novela lleva por título Andá cantale a Gardel y fue publicada en 1970.

${ }^{4}$ Martín Fierro. Gaucho, héroe, mito. Buenos Aires, Plus Ultra, 1967 y Prólogo y Notas a Hernández, José. Martín Fierro. Barcelona, Nauta, 1969.

5 "Ciro Alegría como fundador de la realidad hispanoamericana". Acta litteraria (Budapest), N XVII, 71-92, 1975.

6 "La obra de J. M. Arguedas y la sociedad andina. Interpretación de su praxis literaria como praxis social". Eco (Bogotá), № 162, 592-620, 1974. 
productor-lector-urbano que la creaba" (La Historia, 11). Luego, en la elaboración común de ciertas formas, lenguajes y convenciones como

...la aplicación de una forma de configuración estética para juzgar esa totalidad social y, lo que era más importante todavía, tomando como medida valorativa la perspectiva de las clases tradicionales que sufrían el proceso destructivo de transformación; la tendencia a reproducir el horizonte anímico y la vida cultural de los sectores tradicionales, convertidos en protagonistas centrales del mundo literario; el tono elegíaco predominante que determinaba el lenguaje del relato; la incorporación de la naturaleza a la vida social de aquel estrato popular (11).

Y, por último, elementos comunes fundamentados en los modos de recepción y apropiación de otras tradiciones culturales, como "la persistencia de aquellas características estilístico-formales que ya habían sido descritas por Lukács para dar cuenta de las 'leyes de la épica', que controlaban la estructura y las formas de configuración de la novela histórica en su periodo realista europeo y que, sin embargo, perduraban hasta el presente en América Latina" (11). "Leyes de la épica" que, por cierto, desde hacía más de un siglo habían cesado de aparecer en las diversas formas literarias de los países industriales, siendo inclusive algunas de ellas, así al menos lo asumía Losada, desconocidas en las literaturas europeas modernas. Esta serie de hechos evidenciaban la existencia de lógicas específicas latinoamericanas que, de este modo, le asignaban diferencialidad a los fenómenos culturales del continente y que Losada se propone sistematizar por medio de la elaboración de un riguroso modelo hipotéticodeductivo, debido a que el ensayo, "las más de las veces simplificador y voluntarista", de acuerdo con sus propias palabras, no podría dar cuenta, de un modelo de investigación estratégico, fundamentado en una serie de pautas lógicas.

Esta hipótesis investigativa surgida durante su estancia en la Universidad Nacional Mayor de San Marcos, entre 1971 y 1975, la continuaría desarrollando de manera compulsivamente metódica durante su permanencia de un año como profesor visitante en el Institute of Latin American Studies de la Universidad de Texas at Austin, en 1976, y partir de finales de aquel año durante su periplo europeo en las universidades de Erlangen/Nuremberg (1976-1978) y luego en el Lateinamerika Institut de la Universidad Libre de Berlín. En esta última casa de estudios crearía, en 1982, la AELSAL, Asociación para el Estudio de las Literaturas y las Sociedades en América Latina, institución que organizará importantes congresos (Giessen, 1982; Travers, 1983, 1984 y 1986) en los que se expondrán los sucesivos avances de la investigación para cuyo fin se torna indispensable la elaboración de un modelo historiográfico sistemático: la Historia Social de las Literaturas Latinoamericanas. Proyecto en el que trabajó hasta su muerte, producida en un accidente aéreo ocurrido próximo a La Habana, el 19 de enero de $1985^{7}$.

\footnotetext{
${ }^{7}$ Con posterioridad a la muerte de Losada, el Grupo de Berlín continuará desarrollando el proyecto de Historia Social de las Literaturas Latinoamericanas, publicando, en los años inmediatamente posteriores a su deceso, actas de congreso, secciones especiales de revistas, como el № 30 de la Revista de Crítica Literaria
} 


\subsection{Supuestos hipotéticos y categorías de análisis}

El desarrollo sistemático del modelo de historización propuesto por Alejandro Losada se basa en tres supuestos hipotéticos. A partir de cada uno de ellos, el investigador argentino propondrá una serie de categorías de análisis que guiarán su programa investigativo. El primero de los supuestos que controla el trabajo de Losada surge precisamente de las conclusiones de su etapa de trabajos monográficos y guarda relación con "la precomprensión de la literatura hispanoamericana como un particular, distinto del europeo" (La literatura, 7). Para fundamentar teóricamente este presupuesto Losada elabora los conceptos de Especificidad y Sujeto productor de cultura. La Especificidad es la hipótesis más general de la investigación losadiana. Con ello se pretende marcar una ruptura con aquella tradición remota en los estudios literarios latinoamericanos que intentaba observar las manifestaciones literarias del continente como reproducciones de las literaturas metropolitanas. El recorrido histórico del proyecto de Historia Social de las Literaturas Latinoamericanas establecerá que el objeto de estudio de aquella vertiente tradicional constituye tan solo una de las diversas manifestaciones literarias del continente, la que se encuentra coexistiendo con otros diversos modos que corresponden al resultado de un proceso particular de desarrollo social latinoamericano. En el pensamiento de Losada, el indicador de Especificidad se encuentra estrechamente ligado a la superación y liquidación de la herencia colonial.

Para dar cuentas de las especificidades de este proceso, es que Losada acuña el término de Tipo social productor de cultura, ya no identificado a un autor individual, sino que al grupo social al que cada sujeto se articula. De este modo, y en diferentes y diversos momentos históricos, los sujetos productores de cultura latinoamericanos han respondido a circunstancias concretas del continente con formas culturales particulares y novedosas, las que se encuentran mediatizadas tanto por elementos metropolitanos, como por otros elementos de otras culturas que han sostenido su presencia en nuestro continente.

El segundo supuesto hipotético que subyace al modelo con que Losada pretende historizar la relación entre Literatura y Sociedad en América Latina es la "Convicción de que esa especificidad literaria tiene su origen en fenómenos histórico-sociales globales que han de ser determinados por la investigación" (La literatura, 7). En este plano de la discusión Losada introduce los conceptos de Momentos y Periodos. Ambos son complementarios e intentan superar la homogeneidad espacial y social de la periodización historiográfica literaria tradicional. Los Momentos corresponden a extensos lapsos, con diversas características de desarrollo social. Los Momentos, a su vez, podían ser formativos y constitutivos. Los primeros correspondían a "todo el espacio temporal donde van apareciendo los rasgos que caracterizan a una formación social" (Morales Saravia, Homenaje, 11), y los segundos a "todo el espacio temporal

Latinoamericana (1987), o libros de homenaje a su fundador y organizador, como el volumen Homenaje a Alejandro Losada (1986), editado por José Morales Saravia. 
donde la sociedad se vuelve a reformular" (11), por lo que cabría considerar dentro de este Momento a los procesos de reestructuración oligárquica, liquidación de herencia colonial y modernización capitalista. Al interior de estos recortes temporales extensos se distinguían los Periodos, lapsos más restringidos y que también cumplían la función de marcar diferencias específicas de desarrollo dentro de cada subregión del continente.

El último supuesto hipotético que controla la construcción del modelo se refiere a la "concepción particular acerca de la naturaleza histórico-social del fenómeno literario en América Latina". En este sentido, y en una posición dominante de la crítica latinoamericana desde el desarrollo de la sociología en el continente, solo se demostrará la comprensión de un nuevo fenómeno literario en las sociedades latinoamericanas cuando se logre reproducir cuál ha sido el proceso histórico-genético de formulación del nuevo paradigma estético-cultural que lo sustenta ${ }^{8}$. De este modo, la emergencia de un nuevo conjunto literario se articula en varios niveles: con la formación de un nuevo tipo social productor de cultura, con el establecimiento de una nueva forma de relaciones con los demás actores sociales, con la adopción de una nueva actitud respecto de la cultura nacional dominante y, simultáneamente, con la cultura y los lenguajes literarios internacionales. Persiguiendo esta cadena de articulaciones se podrá interpretar la aparición de un nuevo conjunto de obras literarias, como el resultado último de ese proceso social. Lo que en definitiva se traduce en la reconfiguración de la disciplina en torno a dos objetos: un objeto material, el cual es el conocimiento de las especificidades de las sociedades latinoamericanas, y un objeto formal; el conocimiento específico de las literaturas del continente.

Para este tercer supuesto hipotético Losada construye las categorías de análisis de Espacio social, Modo de producción cultural, Paradigma estético-cultural. El primero es entendido por Losada como "una totalidad social constituida por las relaciones contradictorias entre tres sociedades articuladas: Europa/USA como centros hegemónicos, las sociedades tradicionales "interiores" en la región latinoamericana y las ciudades en proceso de modernización" (La historia, 22). Al interior de este Espacio social latinoamericano surgen diversas estrategias para otorgar sentido de pertenencia a la sociedad y, con ello, diversas funciones sociales cumplidas en aquellas coyunturas específicas (Modos de producción cultural), así como apropiaciones y transformaciones de formas, lenguajes, géneros y convenciones procedentes de distintos movimientos culturales (Paradigma estético-cultural). A partir de este análisis surgen los grandes Sistemas literarios de las literaturas latinoamericanas, propuestos en el modelo de Alejandro Losada: las literaturas dependientes (tipo social productor de cultura en situación supeditada con los grupos hegemónicos), marginales (tipo social productor de cultura desvinculado de los problemas de su respectivo espacio social, como pueden ser

\footnotetext{
${ }^{8}$ Por ejemplo, y sin ir más lejos, en una encuesta sobre el estado actual de la crítica literaria en Latinoamérica realizado por la revista Texto Crítico en su número 6 (enero-abril de 1977) Cornejo Polar señalaba como una de las prioridades de la misma: "Mientras no sepamos cómo funciona socialmente la literatura latinoamericana será prácticamente imposible comprender con rigor el sentido de su desarrollo histórico y hasta sus manifestaciones textuales concretas" (La crítica literaria, 11).
} 
los casos del "modernismo" y el "vanguardismo"), autónomas (donde los tipos sociales creadores de cultura de modo independiente crean sus propios modos y paradigmas culturales, oponiéndose con y a partir de ellos a su respectivo espacio social, como el "romanticismo"), social-revolucionarias (espacios sociales en crisis en el interior de los cuales los tipos sociales productores de cultura crean proyectos alternativos, como en los casos del "indigenismo", el "negrismo", la "narrativa social" en sus diferentes versiones geoculturales) y, por último, las literaturas internacionalizadas (los tipos sociales productores de cultura se han desplazado de su lugar de origen a otros espacios sociales metropolitanos, asumiendo problemáticas de allí, y alcanzando con ello nuevos públicos y por esto nuevas funciones).

\subsection{Potencial interpretativo de cultura y niveles de análisis}

Como él mismo lo asume en varios de sus trabajos de comienzos de los ochenta, Losada construye su modelo de historización hipotético-deductivo basándose en cuatro niveles de análisis: a) Nivel de los supuestos teóricos básicos; b) Nivel estratégico; c) Nivel metodológico; y d) Nivel sistemático. Correlativos a estos cuatro niveles de análisis pueden distinguirse aún otros dos, presentes de modo transversal y permanente a lo largo de todo el proceso: e) Nivel polémico, de acuerdo a como lo define Morales Saravia, al que habría que agregar f) Nivel autocrítico, característico del trabajo losadiano. No obstante, antes de pasar a explicar en qué consisten cada uno de ellos, y para comprender, desde luego, el sentido de la formulación de esta serie de niveles, se hace necesario explicitar el marco que posibilita esta opción metodológica. Ese marco es el potencial interpretativo de la versión antropológica del concepto de Cultura 9 .

La versión antropológica de Cultura comienza a circular de modo sistemático en América Latina alrededor de las décadas de 1920 y 1930, a partir de los trabajos señeros de Gilberto Freyre, Sérgio Buarque de Holanda, Fernando Ortiz, entre otros, conocedores de las investigaciones de Franz Boas o Bronislaw Malinowski. No obstante, como en toda historización de la circulación social de los discursos, existen antecedentes previos de la recepción, o al menos del conocimiento, del concepto antropológico de cultura. En el caso latinoamericano resultan ineludibles en este sentido, los actualizados y contingentes conocimientos que tenían de las propuestas científicas europeas — desde la museología a la antropología, de la fisiología a la psicología - a fines del siglo XIX Silvio Romero en la América Lusa, y Eugenio María de Hostos en la América Hispánica. Más allá de estos episodios ineludibles, para Ángel Rama básicamente son tres las aportaciones que la versión antropológica de cultura realizó para una comprensión más exhaustiva de las literaturas latinoamericanas.

\footnotetext{
${ }^{9}$ Cabe señalar que las reseñas biográficas sobre Alejandro Losada marcan como hecho influyente en el desarrollo antropológico de su trabajo, el encuentro con el reconocido antropólogo especializado en la zona andina, Richard Schaedel, acontecido durante la permanencia de Losada como profesor visitante en el Institute of Latin American Studies en Austin, Texas, durante 1976.
} 
El texto en el que Rama expone estas tres aportaciones, "La literatura en su marco antropológico", es significativo porque, además de ser su último texto escrito y leído antes de morir ${ }^{10}$, debe también considerarse que Rama fue el crítico latinoamericano que más historizó y problematizó las implicancias y significaciones que representó el concepto de cultura al interior del movimiento de renovación crítica latinoamericana a partir de la década de $1960^{11}$. Miradas retrospectivamente, fueron efectivamente estas aportaciones las que, en articulación con el propósito de construir una metodología derivada de los rasgos históricos específicos latinoamericanos, de modo residual impactaron sobre elementos dominantes de la década de 1960 (como el auge de la Nueva Narrativa Latinoamericana y las Ciencias sociales, el concepto de Ideología, entre otros) empujando a una renovación profunda y de carácter colectivo en la Crítica Literaria Latinoamericana. Por ende, también, un par de décadas más tarde, la sedimentación de estas aportaciones, en el marco de emergentes contextos de políticas geoculturales, van a provocar amplios debates y enjuiciamientos.

En primer lugar, afirma el intelectual uruguayo, una de las mayores potencialidades interpretativas a las que contribuye la perspectiva culturalista es a "la afirmación de la legitimidad, independencia y autosuficiencia de cualquier cultura, que debía medirse de acuerdo con sus propios patrones y no ajustarse a los que regían otras culturas (...) y la afirmación de la constitutiva organicidad que la sostiene y autentifica" (Rama, La literatura, 163). Esta interpretación sobre los procesos particulares llevados a cabo por cada sociedad conduce a enfatizar el rol constituyente que desempeñan las condiciones y situaciones concretas de cada lugar en la recepción, apropiación y transformación de ideas, movimientos, formaciones y tradiciones provenientes de otras sociedades, y por ende, constituidas en otras condiciones y situaciones específicas. Como lo planteaba Alejandro Losada:

El impacto que tendrán los movimientos ideológico-culturales del siglo XIX europeo no será el mismo, por ejemplo, en sociedades esclavistas como el Imperio del Brasil, o en sociedades con una mayoría indígena perteneciente a una antigua cultura, como las andinas, que en espacios sociales en donde recién se estaba consumando el proceso de ocupación de la tierra, como en el Río de la Plata. Dentro de estos concretos cuadros de relaciones, la producción de literatura debe cumplir nuevas funciones sociales que, por ser diferentes de las que

\footnotetext{
${ }^{10}$ Bien digo, "escrito y leído". Ya que este texto fue presentado en las "Segundas Jornadas de Sociología de la Literatura", encuentro realizado en Madrid, entre el 16 y el 18 de noviembre de 1983. El otro de sus últimos dos textos, "De la concertación de los relojes atlánticos", preparado para el "Primer encuentro Hispanoamericano de la Cultura", organizado en Bogotá entre el 28 de noviembre y el 2 de diciembre de 1983, no llegaría a ser leído. El día antes al inicio del encuentro, ocurrió el fatídico accidente aéreo en que Rama y su pareja Marta Traba morirían. Este último texto aparecería de forma póstuma el 10 de diciembre de 1983 en el № 319 del suplemento Sábado del periódico mexicano Uno más uno.

${ }^{11}$ Aparte del citado "La literatura en su marco antropológico", la recepción y apropiación de Cultura en su versión antropológica ocupa un lugar importante en otros ensayos de Rama como "Sistema literario y sistema social de Hispanoamérica" y "Un proceso autonómico: de las literaturas nacionales a la literatura latinoamericana".
} 
caracterizan a las literaturas europeas, determinan la transformación de aquellos lenguajes internacionalizados, organizándose procesos que son específicos de estas subregiones (Articulación, 228-229).

Asumir, por tanto, el rol activo y constituyente de las condiciones concretas de cada una de las diversas situaciones sociales implicó cambiar una serie de presupuestos metodológicos sobre el estudio de las literaturas y las culturas latinoamericanas con los cuales habían trabajado las tradiciones selectivas previamente dominantes. Entre ellos, sin duda el más determinante, era invertir el sentido de aquel presupuesto metodológico que exponía como evidencia la deficitaria inserción de las producciones literarias y culturales latinoamericanas en el marco de la internacionalización:

De tal manera que el presupuesto metodológico que destaca la internacionalización de estos fenómenos no implica que haya que observarlos como si fueran solamente pasivos y dependientes de la difusión externa; sino, sobre todo, hay que aislar la manera en que cada uno de los sujetos sociales productores de literatura en cada subregión se apropia activamente de aquellos movimientos y los transforma para que cumplan otras funciones, en otro contexto de relaciones y con otros actores sociales (Losada, Articulación, 229).

Por otra parte, el cambio de perspectiva en la apropiación de elementos externos, desde una posición pasiva hacia una activa, da paso a una segunda aportación a la que constituye la perspectiva culturalista, esta vez enfocada en la interrelación de sus elementos internos, la que es fomentar "una visión estructurada de las culturas, subrayando la estricta correlación de sus diversos términos (lengua, creencias, formas sociales, artes) en una dinámica fuertemente interdependiente" (Rama, La literatura, 164). La diferencia fundamental es que comienzan a desarrollarse programas críticos que intentan dar cuenta del fenómeno literario latinoamericano entendiendo lo atinente a la sociedad no ya como variable independiente del proceso, sino que complementariamente determinada también por la variable literaria. Es decir, que la autonomía de la cultura latinoamericana no surge en su confrontación a los modelos metropolitanos, sino que a partir de la particularidad cultural de América Latina, por lo que la literatura deja de ser percibida como producto de la estructura social, para ser comprendida como resultado de la praxis social, en tanto actividad, producción, y autoproducción, resultado de una serie de relaciones que posibilita, a su vez, otras relaciones que intervienen considerablemente en las prácticas sociales materiales.

Queda en evidencia, entonces, que el modelo de análisis que Losada denomina tradicional solo puede dar cuenta del fenómeno literario latinoamericano como un todo indiferenciado, unitario y estático, como ocurre con la metáfora del mestizaje, las hipótesis de lo real maravilloso o el realismo mágico. Asumiendo, por tanto, la legitimidad, independencia y autosuficiencia de cada cultura, por un lado, y su correlación dinámica e interdependiente, por otra, un modelo que intente dar cuenta de las particularidades y contradicciones de los procesos literarios latinoamericanos no solo debe articular a este último a un contexto internacionalizado, sino que también surge la 
necesidad de articularlo al efecto que producen los fenómenos hegemónicos "sobre cada formación social subregional en cada una de esas etapas. Hay que articular, entonces, los procesos literarios a los procesos de formación y transformación de cada sociedad subregional" (Losada, Articulación, 232). La literatura se asume de este modo como pregunta y respuesta de los fenómenos que condicionan el desarrollo del proceso social-cultural. Su función, en definitiva, se extiende desde la interrogante, previamente dominante, que apuntaba a comprobar si se producían procesos literarios dependientes de los centros hegemónicos, hacia la inclusión de analizar si se producen procesos literarios que intenten "liquidar esa situación dependiente y, o bien sea producido por la misma clase oprimida, o bien pretenda articularse a sus contradicciones y a su movilización". Esta extensión condujo a que formaciones como la literatura indigenista, la literatura de la Revolución Mexicana, el testimonio, entre otras, pasen a ocupar lugares preponderantes en las agendas de investigación a partir de la década de los sesenta.

Por último, y en estrecha relación con las anteriores, la tercera aportación relevante que entrega la adopción de la perspectiva culturalista es "la noción de producción colectiva de la cultura que desarrolló la antropología, a partir del examen de las artes y sobre todo la lengua de las culturas primitivas" (Rama, La literatura, 165). A partir de esta aportación puede comprenderse la noción losadiana de Tipo social productor de cultura ${ }^{12}$. Esta noción de producción colectiva de la cultura, debe mucho su desarrollo y formulación al potente avance que registró el materialismo, sobre todo desde Feuerbach y Marx en adelante con la comprensión de la realidad como la interacción entre la subjetividad humana, la cual es histórica y colectiva, y el mundo material que aquella subjetividad crea a partir de sus actividades. En torno a estas potenciales aportaciones de la versión antropológica de Cultura puede comprenderse cuál es el enmarque en el que Losada desarrolla sus niveles de análisis.

En el marco de esta aportación del concepto antropológico de cultura, Losada construye el primer nivel de su modelo de sistemas literarios como instituciones sociales, el nivel de los supuestos teóricos básicos. Y como puede sintetizarse a partir de la exposición previa, los fundamentos de este nivel reposan en cuatro evidencias investigativas solventadas en los alcances de la especificidad latinoamericana. Estas son: primera evidencia, queda claro que, debido a que se insertan en un universo cultural diferencial, el uso del lenguaje literario que realizan las literaturas latinoamericanas expresa y cumple por ende, también, otras funciones, por lo que en definitiva se trata de un lenguaje literario distinto. Segunda evidencia, se asume, de igual modo, que constituimos sociedades distintas respecto de otros espacios geoculturales metropolitanos. Tercera evidencia, en nuestras sociedades y en sus respectivas literaturas, los sujetos productores de cultura tienen diferentes caracteres y cumplen diferentes funciones. Cuarta evidencia: si hablamos de la legitimidad y autosuficiencia

\footnotetext{
${ }^{12}$ A partir de esta tercera aportación, puede comprenderse también el enmarque de otras categorías de análisis culturales de intención colectiva, paralelas a la propuesta losadiana, como "Ciudad letrada", por ejemplo.
} 
de cada cultura, corresponde no analizar más a Latinoamérica como un todo indiferenciado, sino que asumir el estudio de los diferentes procesos genético-históricos para cada subregión del continente. Se trata, en definitiva, de sociedades latinoamericanas y literaturas latinoamericanas. Por ello, Losada plantea el estudio del fenómeno basándose en cinco subregiones de nuestro continente: Cono sur, Pacífico Andino, Brasil, Caribe y Centroamérica y, por último, México.

$\mathrm{Y}$ es a este nivel de los supuestos teóricos básicos en que Losada presenta su definición de literatura, construida sobre tres dimensiones. La primera de ellas alude a tomar conciencia de que la literatura y la cultura surgen a partir de una serie de instancias: el proceso de producción, el producto y la relación del sujeto productor tanto consigo mismo como con la sociedad. A esta interacción Losada lo llama la Praxis social. Es debido a esta interrelación que el Tipo social productor de cultura ya no puede entenderse como referente a un autor individual, sino que al grupo social al que cada sujeto se articula. Sin embargo, la literatura no solo surge como el resultado de una serie de relaciones, sino que también ella, a la vez, posibilita otro conjunto de relaciones sociales, como por ejemplo:

...cada vez que se estabiliza una cultura urbana institucionalmente, el sistema literario está referido predominantemente al estrato especializado en la cultura. Y a partir de la consolidación de este grupo social, es posible observar el fenómeno literario como una manera de establecer relaciones sociales con los otros tres actores sociales también organizados institucionalmente: la masa popular, la élite dominante del poder económico y político y, finalmente, los centros hegemónicos externos (Losada, Articulación, 239-240).

Es a este respecto que, además, Losada entiende a la literatura como Institución social. Aún hay un tercer nivel por el cual Losada comprende el concepto de literatura, además de los anteriores de Praxis e Institución, el que refuerza el carácter colectivo de ella y también, por ende, de la cultura. La literatura, en su concepción, también involucra una apropiación del mundo por medio de la palabra, por lo que instituye un sentido, crea un Horizonte de existencia —el concepto es de Heidegger-, desde el cual se comprenden los comportamientos, sentimientos y modos de aprehender y situarse en el mundo de cada sujeto y cultura. De este modo, el Horizonte de existencia fundamenta cómo los diversos sujetos y grupos sociales, por medio de la literatura, otorgan sentido tanto a su existencia como a su relación con el mundo ${ }^{13}$.

El segundo nivel de análisis en el modelo losadiano que surge a partir de las aportaciones de la versión antropológica de cultura, es el nivel estratégico. A este nivel

\footnotetext{
${ }^{13}$ Acota Patricia D’Allemand: Este concepto heideggeriano, señala Losada, remite a la discusión sobre la "subjetivización" del sentido en la edad moderna: si en la antigüedad clásica y en la Edad Media regía una totalidad de sentido que trascendía a la subjetividad de los individuos, en la edad moderna este sentido se ha roto obligándolos a "...construir el mundo a partir de la interioridad...” (Hacia una crítica, 122). Esta fundamentación histórica de la subjetividad refuerza la necesidad de un concepto como cultura para la Modernidad.
}

292 
se construye el corpus de análisis, basándose en un criterio de representatividad que justifique la conformación de corpus más amplios que excedan la etapa monográfica de investigación. El criterio de representatividad se fundamenta en la elección de textos que debían ser momentos claves dentro de cada proceso histórico-social. La selección del corpus de trabajo según criterios de representatividad se vinculaba estrechamente a la dimensión de los supuestos teóricos básicos. Es decir, que constituían textos representativos aquellos que daban cuenta de nuevas relaciones y funciones sociales, que concretaban nuevas formas estéticas al interior de paradigmas estético-culturales, textos a partir de los que se identificaban especificidades de la cultura latinoamericana ${ }^{14}$. Una vez seleccionados estos textos representativos de cada subregión y de cada paradigma estético-cultural, se procedía a agruparlos sistemáticamente en conjuntos literarios "según sus semejanzas con respecto a los sujetos sociales productores, los paradigmas estético-culturales que estos elaboraban y las funciones que pretendían cumplir" (Morales Saravia, Homenaje, 11).

En este nivel también operaban los problemas relativos a la periodización. Para ello, Losada a lo largo de la investigación llegó a establecer dos categorías complementarias para superar los problemas de simultaneidad y diferencialidad con los que había operado la tradición historiográfica latinoamericana previamente dominante. Estas dos categorías son las ya aludidas de Momentos y Periodos.

El siguiente es el nivel metodológico. Este es el nivel propiamente tal de análisis de los conjuntos literarios. Para ello, como reconoce José Morales Saravia, se procedía a trabajar en varias instancias:

Se preguntaba por el sujeto social productor de estos conjuntos. Esto implicaba evitar procedimientos biografistas o superar el fetichismo del "texto". Se analizaba qué paradigma estético-cultural elaboraba ese sujeto social: temas, lenguajes, géneros, formas, estilos así como modelos de gusto y mecanismos de recepción de otros fenómenos culturales. Se describía las funciones que pretendían cumplir los sujetos sociales productores elaborando esos paradigmas, sean estas de coopción, elusión, cambio, etcétera. Y se veía en qué espacios sociales se movían esos sujetos productores para los que elaboraban esos paradigmas que cumplían funciones determinadas. Todas estas instancias interrelacionadas en el análisis eran subsumidas bajo la noción de modo de producción (Homenaje, 11).

De esta manera, en un último nivel, el nivel sistemático, se integraban resultados alcanzados por el análisis de los diversos modos de producción en Sistemas literarios, los que pasaban a relacionarse entre sí, con el objeto de ser expuestos en una

\footnotetext{
${ }^{14}$ Por ejemplo, en el trabajo El Surgimiento del Realismo Social en la Literatura de América Latina, Losada expresará respecto de la inclusión de "El Matadero" de Esteban Echeverría dentro de este paradigma estéticocultural: "El Matadero constituye el primer texto literario que concreta en una nueva forma estética las funciones que el grupo autónomo había asignado a la literatura cuando anunció su nuevo proyecto estético-cultural, dando por resultado la aparición del realismo social en el proceso de la literatura hispanoamericana" (44).
} 
formulación final. Correlativos a estos niveles de análisis estratificados, persistían, en proyecto de Historia Social de las Literaturas Latinoamericanas de Losada otros dos niveles igualmente constitutivos del modelo hipotético-deductivo. Por una parte, un nivel que Morales Saravia califica de Polémico, debido a que en cada instancia de elaboración de su propuesta, Losada se encuentra estableciendo constantes diatribas con los modelos con los que trabajó la tradición previa historiográfica latinoamericana. Aunque estas tomas de posición no solo enfrentan a la tradición previa, indudablemente también a las corrientes contemporáneas al desarrollo de la Historia Social de las Literaturas Latinoamericanas.

Un segundo nivel correlativo que debe distinguirse como constitutivo del modelo de análisis losadiano es el nivel autocrítico, sumamente característico de su investigación, $\mathrm{y}$, por lo demás, inherente a la estructura circular de todo pensamiento sistémico. A la manera de los textos aporéticos de la filosofía helénica clásica, Losada avanza en su elaboración sometiendo permanentemente a examen sus formulaciones y conclusiones previas. Sus exposiciones podrían considerarse especies de diarios o bitácoras de investigación, por mostrar todas las instancias del proceso constructivo, pertenecientes tanto a lo andado como a lo desandado. En la reseña que Antonio Cornejo Polar escribe sobre Creación y praxis (Revista de Crítica Literaria Latinoamericana $\mathrm{N}^{\circ}$ 5, 1977), este nivel correlativo ocupa un lugar recurrente de atención. Allí, el crítico peruano no deja de destacar lo apasionante que resulta para los lectores esta característica, en la que Alejandro Losada "desdobla su discurso para dar razón de la materia que quiere esclarecer, pero también, y con el mismo y hasta mayor énfasis, para poner en cuestión los principios teóricos y los recursos metodológicos que va empleando para lograr este esclarecimiento" (Cornejo, Creación, 130), resultando ser "un apasionante itinerario científico, algo así como la revitalización de la aventura del conocimiento que no cesa de preguntarse por su propia validez" (131). Aunque, por supuesto, y en su característica ironía fina y aguda, Cornejo Polar tampoco desatiende lo confuso que puede terminar siendo este procedimiento en términos de exposición: "No solo determina un exceso de reiteraciones, correspondientes al afinamiento de determinados conceptos, sino que, a la larga, crea confusiones hasta en el lector más atento" (131). La confusión más sensible que crea el nivel autocrítico de Losada en aquella lectura de Cornejo Polar es "el tratamiento de la narrativa de José María Arguedas". Primero, porque al crítico peruano no le queda claro "hasta qué punto los enfoques posteriores al primer análisis modifican los términos de este", los que, además, a juicio de de quien reseña, son "erróneos" (131). Cornejo los considera de tal forma porque, "Allí se juzga a Arguedas no tanto por lo que significa su obra narrativa, vista algo esquemáticamente, cuanto, más bien, por lo que no representó", en una interpretación en la que "Casi se le llega a exigir otra materia y una distinta perspectiva" (131). Este comentario de Cornejo revela uno de los grandes problemas del modelo teórico de Losada y, aunque sin aludirlo directamente, el crítico peruano retomaría aún en otro trabajo dedicado a evaluar el enfoque sistémico aplicado a las literaturas latinoamericanas. En lo que sigue, y para finalizar, propondré un fundamento general 
que intente explicar no solo esta crítica al trabajo de Losada, sino que aún otra; la que realiza Patricia D'Allemand en Hacia una crítica cultural latinoamericana.

\section{De la aventura del conocimiento a la aventura americana (del lector)}

Sin establecer la relación de manera directa, de hecho sin siquiera mencionar el nombre de Alejandro Losada aunque el título del trabajo es explícito, el problema que Antonio Cornejo Polar desarrolla en su artículo "Los sistemas literarios como categorías históricas. Elementos para una discusión latinoamericana" se vincula solapadamente con lo ya expuesto en su reseña de 1977. En aquel artículo, Cornejo argumenta que el principal problema que enfrenta el enfoque sistémico como método para la historiografia literaria latinoamericana es su débil consideración de la dimensión temporal. Una concepción del tiempo que Cornejo califica de abstracta, única, armoniosa, por ende hechiza, en la medida en que se rige por la imposición temporal del progreso. En definitiva, un "tiempo ontológico" que Losada asume desde la fenomenología alemana. Lo que Cornejo reclama allí es una de las demandas centrales de su trabajo, y por ello mismo, una de sus principales aportaciones al análisis crítico de las literaturas latinoamericanas. Me refiero al reclamo por las múltiples temporalidades "con que se trama, sin pausa, la aventura americana" (Cornejo, Sistemas literarios, 25). De la "aventura del conocimiento" del 77 a la "aventura americana" del 88, el gran problema que Cornejo les endosa a los sistemas literarios como categorías históricas es que reducen en un único tiempo homogéneo (y hegemónico que por tanto llega a "exigir otra materia y una distinta perspectiva") a todas las manifestaciones culturales disímiles que habitan el espacio social latinoamericano, un tiempo que Cornejo define como geológico más que histórico.

En efecto, la categoría de "sistema literario" significa para la crítica literaria latinoamericana del periodo, en el arco teórico sobre el concepto que recorre desde las elaboraciones respectivas de Antonio Cándido, Ángel Rama hasta Alejandro Losada, un gran avance en la dimensión metodológica, aunque no así en la dimensión epistemológica. En su intento por superar los problemas relativos a la sucesividad, simultaneidad y diacronicidad de las literaturas del continente, la categoría en cuestión corrige "los vicios de la historiografia, pero — pésimo negocio - casi nos quedamos sin historia" (20), como enuncia Antonio Comejo Polar en "Los sistemas literarios como categorías históricas". Las críticas desplegadas en aquel ensayo por el maestro peruano apuntan a una dirección similar a las que Haroldo de Campos esbozara en su crítica a la Formaçao da literatura brasileira de Antonio Cándido, planteamiento matriz de la categoría de "sistema" en la crítica latinoamericana. Por ende, la categoría de "sistema literario", desde Cándido a Losada, constituye un replanteamiento de problemáticas historiográficas con énfasis espacial mas no temporal. En esta sublimación de las diversas y entreveradas temporalidades subsumidas en el tiempo hegemónico de la modernización continúa repitiéndose un trazado rectilíneo que solapa las disrupciones, los accidentes, los márgenes, encarnados a este respecto en las manifestaciones literarias tanto populares como indígenas. El mayor detrimento de este trazado rectilíneo encubridor de diferencias 
es que no proponía ninguna crítica de base al discurso histórico de la modernidad. Más allá de la revalorización de ciertas producciones literarias latinoamericanas, se las seguía insertando en una sola gran narrativa, con un solo y marcado ritmo histórico y que, por ende, no erradicaban del todo las jerarquizaciones impuestas por las literaturas cultas y urbanas, el clasismo nacionalista, la pretensión objetivista y la teleología inmanente que venían desplegándose, de modo dominante, en la historiografía literaria latinoamericana desde el momento mismo de su emergencia.

Vuelvo a insistir, la reconsideración que efectúa Losada en su modelo sobre el problema de la simultaneidad que él atribuía a la tradición dominante previa de la historiografia literaria latinoamericana es solamente espacial más no temporal. En síntesis, no se establece una demarcación sobre los distintos tiempos con los que trabajan tanto las literaturas dependientes, como las marginales, tanto las internacionalizadas como las social-revolucionarias. El fundamento general de esta crítica, y que considero como la principal problemática que se desprende de la propuesta losadiana, es que esta sigue operando en una lógica "sujeto/objeto", la que, como dice Chaitin, ha "dominado la filosofia occidental desde Bacon y Descartes" (162) en adelante, hasta con seguridad las primeras décadas del siglo XX. Desde esta lógica, solamente pueden reconocerse los cambios de estadios o variaciones - Momentos y Periodos - , pero no permite reconocer las interrelaciones dinámicas e internas de cada proceso cultural. No permite reconocer, entonces, el indesmentible hecho de que ninguna manifestación o expresión cultural pertenece por completo a un solo sistema o formación. En la práctica, y producto de la continua interrelación social, todo hecho de la cultura debe su constitución a elementos de variada procedencia. Por lo general siempre es un trabajo entrelazado de múltiples "sistemas", en muchos casos, inclusive, antagónicos. Esta consideración también explicaría el argumento circular que, de acuerdo con Patricia D'Allemand, controla el análisis losadiano. Expresa D'Allemand: "los escritores "marginales", que son reaccionarios, seleccionan discursos reaccionarios y por eso producen proyectos reaccionarios", lo que conduce en definitiva a perder de vista, el presupuesto básico del mismo Losada de que "en todo proceso de producción cultural en América Latina habría "un proceso de selección y transformación" de los materiales tomados tanto del polo europeo como del local tradicional" (Hacia una crítica, 109). Una lógica "sujeto/objeto", por tanto, siempre conllevará, explícita o implícitamente, un sentido de dependencia, de subalternidad, la que no solo propicia un argumento circular, sino que también, aún más importante, una relación hegemónica circular.

Las interrelaciones dinámicas internas constituyentes de cada proceso social solo pueden ser interpretadas por medio de una lógica que Chaitin define como lógica del Yo/Tú o también sujeto/sujeto, cuyas formulaciones teóricas mayormente reconocidas se encuentran en conceptos como Alteridad y Dialogismo, pero también en otras tantas, como la, nunca muy bien comprendida, Estructuras de sentimiento de Williams, la reapropiación que hace Hall sobre Articulación de Laclau, Habitus de Boudieu, por mencionar algunas, y, en el plano latinoamericano, presente también, y sin ir más lejos, en las propuestas de Cornejo Polar de Heterogeneidad y Sujeto migrante. De ningún 
modo, por cierto, esta lógica Yo/Tú involucra una regresión metodológica a trabajos monográficos. Antes bien, de lo que se trata es evidenciar las aporías de aquel pensamiento sistemático y riguroso dominante del pensamiento occidental por medio de las relaciones sociales efectivas de los sujetos y sus circunstancias en el continente americano. En su admirable aventura del conocimiento, Losada optó por una determinación objetiva del fenómeno, antes que por un encuentro dialógico con las diversas subjetividades, siendo este el principal problema que enfrentó tanto su investigación como las consecuencias de ella ${ }^{15}$.

La respuesta no venía dada por construir nuevas sistematizaciones y periodizaciones, aunque sean propias, sino que por sobre todo tomar conciencia de las contradicciones y las paradojas de aquellas mismas operaciones. Como expresa Cornejo en el citado artículo de 1988: "Después de todo, la polifonía bajtiniana solo es enriquecedora cuando las voces de los otros preservan su tono y temple discordante" (23). A una advertencia similar llegaba Ángel Rama en su intervención en el encuentro de especialistas de Campinas de 1983 (del 3 al 6 de octubre), centrado en discutir el problema de la periodización con el objetivo de construir una nueva historia de las literaturas latinoamericanas, y que luego aparecería publicado dos años después como La literatura latinoamericana como proceso:

Llego a esta convicción que me parece fundamental: que la comparación se desprenderá del volumen, del libro que hagamos, pero no de cada uno de los estudios en particular. Que cada uno de los estudios en particular examinará problemas muy concretos en el Brasil, en Hispanoamérica, en las Antillas. La visión comparativa la hará el lector, la reconstruirá el lector viendo la totalidad de este volumen y observando las experiencias literarias que se nos analizan de una zona a otra zona: de México, del Río de la Plata, de la zona paulista (Rama, Algunas sugerencias, 67 , cursiva es mía).

Porque en definitiva, una lógica sujeto/sujeto solo cobra sentido y coherencia cuando se extiende, consecuentemente, como desafio crítico y comprometido, hacia los lectores.

Universidad Andrés Bello*

Fernández Concha 700, Las Condes, Santiago (Chile)

hugo.herrera.pardo@gmail.com

\footnotetext{
${ }^{15}$ Por ejemplo, expresa mucho sobre las consecuencias de esta encrucijada el hecho de que Losada preste escasísima atención e importancia a la producción cultural colonial, justamente en paralelo con las mismas fechas en que este campo de estudio experimenta una reconfiguración de amplísimas dimensiones.
} 


\section{OBRAS CITADAS}

Chaitin, G. "Otredad. La literatura comparada y la diferencia", en: Vega, M.J y Carbonell, N. La literatura comparada: principios y métodos. Madrid: Gredos, 1998.

Cornejo Polar, A. "Losada, Alejandro: Creación y praxis. La producción literaria como praxis social en Hispanoamérica y el Perú". Revista de crítica literaria latinoamericana 5 (1977): 130-132.

__ “La crítica literaria, hoy”. Texto Crítico 6. México: 1976: 6-36.

— "Los sistemas literarios como categorías históricas. Elementos para una discusión latinoamericana". Revista de crítica literaria latinoamericana 29 (1988): 19-25.

D'Allemand, Patricia. Hacia una crítica cultural. Berkeley-Lima: Latinoamericana editores, 2001.

Losada, A. "Los sistemas literarios como instituciones sociales en América Latina". Revista de crítica literaria latinoamericana 1 (1975): 39-61.

_ "El surgimiento del realismo social en la literatura de América Latina". Ideologies \& Literature 2 (1980): 20-55.

__ La literatura en la sociedad de América Latina. Perú y el Río de la Plata, 18371880. Frankfurt: Vervuert, 1983.

—_ "Articulación, periodización y diferenciación de los procesos literarios en América Latina". Revista de crítica literaria latinoamericana 17 (1983): 7-37.

__ "La historia social de la literatura latinoamericana". Revista de crítica literaria latinoamericana 23 (1986): 21-29.

Morales Saravia, J. (Editor). Homenaje a Alejandro Losada. Lima: Latinoamericana editores, 1986.

Rama, Á. "Algunas sugerencias de trabajo para una aventura intelectual de integración". En: Pizarro, Ana (coordinación). La literatura latinoamericana como proceso. Buenos Aires: Centro editor de América Latina, 1985.

__ "La literatura en su marco antropológico". En Literatura, cultura, sociedad en América Latina. Montevideo: Trilce, 2006. 